\title{
Rursus
}

m Ruksus

Poiétique, réception et réécriture des textes antiques

$6 \mid 2011$

Relire, récrire, prolonger. Adaptations gréco-latines

\section{La Fontaine, l'Ancien et le Moderne. Rupture et continuité humanistes}

Anna Jaubert

\section{OpenEdition}

1 Journals

Édition électronique

URL : http://journals.openedition.org/rursus/529

DOI : $10.4000 /$ rursus.529

ISSN : 1951-669X

Éditeur

Université Nice-Sophia Antipolis

Référence électronique

Anna Jaubert, "La Fontaine, l'Ancien et le Moderne. Rupture et continuité humanistes », Rursus [En ligne], 6 | 2011, mis en ligne le 09 février 2011, consulté le 10 décembre 2020. URL : http:// journals.openedition.org/rursus/529; DOI : https://doi.org/10.4000/rursus.529

Ce document a été généré automatiquement le 10 décembre 2020.

Rursus 


\title{
La Fontaine, l'Ancien et le Moderne. Rupture et continuité humanistes
}

\author{
Anna Jaubert
}

Il n'y a pas de culture sans mémoire, mais l'adage facétieux, «la culture est ce qui reste quand on a tout oublié », implique la véritable «innutrition » dans laquelle s'enracine notre humanisme. L'hommage que je veux rendre à Chantal Kircher, spécialiste des langues anciennes, illustrera l'idée de continuité articulée à celle d'évolution, et de transformation, qu'elle a toujours elle-même portée haut. C'est sur La Fontaine, un de nos classiques les plus affectionnés, que l'attention portera, donnant à réfléchir sur l'étiquette-même de classique. On sait que le fabuliste, devenu académicien en 1683, avait embrassé le parti des Anciens dans la célèbre Querelle qui divisa le monde des BellesLettres à la fin du XVIIe siècle ${ }^{1}$. De grands noms s'affichaient dans ce camp : Furetière était une référence, mais c'est Boileauqui surtout avait donné le la avec ses Satires I-VI et VIII-IX (1666-1668), le Traité du sublime de Longin (1674), et bien-sûr L'Art poétique (1674). Rapin, avec les Réflexions sur la Poétique d'Aristote, Racine dans ses Préfaces d'Iphigénie (1675) et de Phèdre (1677) ainsi que La Bruyère et plus tard Fénelon s'illustrèrent aussi dans le débat.

De leur côté, les Modernes avaient pour chef de file Charles Perrault (Le siècle de Louis le Grand, 1687, Les Parallèles des Anciens et des Modernes, 1688, Des hommes illustres qui ont paru en France, 1696-1711), mais avant lui s'inscrivaient déjà dans cette mouvance Georges de Scudéry (Alaric, 1654), Jean Chapelain, (La Pucelle, 1657), Desmarets de Saint-Sorlin (La comparaison de la langue et de la poésie française avec la grecque et la latine, 1670), Défense du poème héroïque, 1675, Défense de la poésie et de la langue française, 1675), et Paul Pellisson ( Relation contenant l'Histoire de l'Académie Françoise, 1672). Dans leurs rangs on compte également Fontenelle (Dialogues des morts, 1683, Digression sur les Anciens et les Modernes, 1687), Saint-Evremond (Sur les poèmes des Anciens, 1686, Sur la dispute touchant les Anciens et les Modernes, 1692), et, à l'aube du XVIII ${ }^{\mathrm{e}}$ siècle, Pierre Bayle (Dictionnaire historique et critique, 1695-1697).

Pour les uns comme pour les autres, l'argumentaire est simple : les Anciens soutiennent une conception de la création littéraire comme imitation des auteurs de l'Antiquité qui, 
selon eux, représentent définitivement la perfection artistique. La Poétique d'Aristote est leur bréviaire. Pour les Modernes au contraire, les œuvres de l'Antiquité ne sont pas indépassables, la création littéraire se doit d'innover dans ses formes, et d'être en phase avec son temps. Mais, on le verra, cette simplicité apparente cache des enjeux beaucoup plus profonds. Ces enjeux traversent les Fables de La Fontaine : ils sous-tendent la posture originale du fabuliste, où l'ancien et le moderne se rejoignent.

Dans sa célèbre Epitre à Huet (1687)², qui officialise sa prise de position en faveur des Anciens, La Fontaine prend soin de déclarer «mon imitation n'est pas un esclavage »: nous le savons, il s'en fallait de beaucoup en effet. A telle enseigne que souvent la critique s'est plu à insister sur la «modernité» du prétendu ancien. L'étude qui suit rappellera quelques traits significatifs de cette modernité. La distanciation, revendiquée, n'était pas anodine. Dans un contexte traversé de tensions, que notre vision rétrospective tend à écraser, La Fontaine proclame avant tout son indépendance d'esprit. Dépassant la pétition de principe, il exemplifie ce qu'il dit, en se réappropriant et en transformant l'héritage des Anciens. Alors, on le verra, l'ancrage et les références puisées chez eux s'avèrent libérateurs.

\section{Le monde des Fables et le retour aux sources}

Certes, l'œuvre de La Fontaine ne se limite pas à ses Fables. Ce sont elles néanmoins qui occupent le premier plan de sa réception, et qui, en l'occurrence, illustreront le mieux les deux visages d'une poétique, marquée à la fois par sa révérence aux Anciens et par une sensibilité très moderne. On sait par ailleurs que sa "Muse galante» s'abreuve plus notoirement à une source moderne, et que ses Contes et Nouvelles notamment, relèvent de l'inspiration grivoise de Boccace ou de l'Arioste. Cet autre ancrage de sa création ne doit pas être négligé, car il éclaire indirectement l'inspiration plurielle à laquelle nous allons nous attacher.

Le genre des fables remonte à l'Antiquité, et La Fontaine dans ses Préfaces s'inscrit officiellement dans la tradition d'Esope, l'inventeur du « bel art » :

Je chante les Héros dont Esope est le père'

Esope, suivi par Phèdre et Avenius, et dont il convient désormais d'accorder le projet avec l'harmonie de la poésie. Car les Fables d'Esope, extrêmement concises, dédaignaient tout ornement: ici, La Fontaine plaide pour une certaine liberté d'adaptation, mais en s'appuyant encore sur une autorité antique, celle de Socrate lui-même. Partant de l'idée que les «Grâces lacédémoniennes ne sont pas tellement ennemies des Muses françaises, que l'on ne puisse souvent les faire marcher de compagnie $»^{4}$, il rappelle que selon Platon, Socrate employa les derniers moments de sa vie à mettre en vers les Fables d'Esope. Et de son côté, pour rendre hommage à celui qu'il met au rang de Sage, il ouvre son premier recueil de Fables par une transcription de Planude, La vie d'Esope le Phrygien.

Ce positionnement clairement affiché dans le péritexte des Fables, se confirme d'emblée dans le contenu du premier recueil : La Cigale et la Fourmi, Le Corbeau et le Renard, puisent leur sujet chez Esope, La grenouille qui veut de faire aussi grosse que le bouf et Les deux mulets chez Phèdre, Le loup et le chien, La génisse, la chèvre et la brebis en société avec le lion chez Esope encore, et ainsi de suite. La fable VII, La Besace, empruntée à Avenius, mais partiellement aussi à Esope et à Phèdre, fait apparaître une autre forme "d'innutrition ", désormais souvent réitérée, mêlant aux acteurs de ce petit monde force personnalités 
mythologiques : c'est Jupiter (appelé aussi familièrement Jupin) qui ouvre la session des doléances pour l'ensemble des créatures, et plus loin, c'est à Junon que se plaint le Paon. Cassandre, Castor et Pollux, Apollon, Mercure, Ulysse, l'olympe et le Parnasse sont couramment pris à témoin. Mais parfois ils surgissent malicieusement au détour d'une caution forcée :

Une Tortue était, à la tête légère,

Qui, lasse de son trou, voulut voir le pays [...]

Deux Canards à qui la commère communiqua ce beau dessein,

Lui dirent qu'ils avaient de quoi la satisfaire :

Vous voyez ce large chemin?

Nous vous voiturerons, par l'air, en Amérique,

Vous verrez mainte République,

Maint Royaume, maint peuple, et vous profiterez

Des différentes mœurs que vous remarquerez.

Ulysse en fit autant. On ne s'attendait guère

De voir Ulysse en cette affaire.

La Tortue écouta la proposition... ${ }^{5}$

Dans la réflexivité accrue du second recueil, l'allusion intempestive à Ulysse est, pour le fabuliste, une manière humoristique de traiter ses habituelles références, en soulignant d'un gros trait leur caractère convenu. Pareille distanciation n'empêche pas ces mêmes références d'apparaître comme parfaitement naturelles dans le vivier des anecdotes. On se reportera au caractère hybride du genre. Un récit, ou apologue, raconte une histoire exemplaire propre à délivrer une leçon de vie : c'est le corps de la fable, la leçon ellemême, explicite ou implicite, intégrée ou détachée, en constitue la morale, qui est son " âme ». Une fable emblématique dans son statut métadiscursif, «Le pouvoir des fables " (VIII, 4), révèle toute la souplesse des transitions entre différents points d'ancrage ${ }^{6}$, ou, si l'on préfère, entre différents « univers de croyance $»^{7}$ du discours. L'événement ici narré possède un fondement historique :

Dans Athène autrefois peuple vain et léger,

Un Orateur voyant sa patrie en danger,

Courut à la Tribune ; et d'un art tyrannique,

Voulant forcer les cœurs dans une république,

Il parla fortement sur le commun salut...

Dans la figure de l'Orateur nous reconnaissons Démosthène qui tentait d'alerter ses concitoyens sur la politique conquérante de Philippe de Macédoine. En vain : le peuple se montrait sourd à sa rhétorique. Il prit alors « un autre tour » propre à le réveiller :

Cérès, commença-t-il, faisait voyage un jour

Avec l'Anguille et l'Hirondelle :

Un fleuve les arrête ; et l'Anguille en nageant,

Comme l'Hirondelle en volant,

Le traversa bientôt. L'assemblée à l'instant

Cria tout d'une voix : Et Cérès que fit-elle?

L'anecdote, inscrite dans un épisode de l'histoire grecque ancienne, accueille, avec l'aventure de Cérès, une inclusion mythologique. Mais il y a mieux encore : ce « conte d'enfant », rapproché de Peau d'Âne, offre à La Fontaine une morale œcuménique :

A ce reproche l'assemblée,

Par l'apologue réveillée,

Se donne entière à l'Orateur :

Un trait de Fable en eut l'honneur.

Nous sommes tous d'Athène en ce point ; et moi-même,

Au moment que je fais cette moralité, 
Si Peau d'âne m'était conté ${ }^{8}$,

J'y prendrais un plaisir extrême.

Une autre fable, emblématique elle aussi de la visée des fables, L'Education, met en regard le double ancrage des références. Les personnages sont deux chiens, deux frères dont les parcours ont divergé :

Laridon et César, frères dont l'origine

Venait de chiens fameux, beaux, bien faits et hardis,

A deux maîtres divers échus au temps jadis,

Hantaient l'un les forêts, et l'autre la cuisine ;

Ils avaient eu d'abord chacun un autre nom ;

Mais la diverse nourriture

Fortifiant en l'un cette heureuse nature,

En l'autre l'altérant, un certain marmiton

Nomma celui-ci Laridon :

Son frère, ayant couru mainte haute aventure,

Mis maint Cerf aux abois, maint Sanglier abattu,

Fut le premier César que la gent chienne ait eu. (VIII, 24).

Certes, un parcours noble justifie une dénomination prestigieuse, à connotation antique : César est un nom propre remotivé, qui vaut titre ; de son côté, Laridon est un nom forgé, moderne, qui, dans ses sonorités, affiche la dégénérescence de la lignée des tournebroches. Mais la morale reste ambiguë : l'évocation héroï-comique des exploits du chien de chasse, rend suspecte l'admiration qui lui est portée, comme elle relativise le mépris pour le chien de cuisine.

Au-delà des déclarations formelles, et des références affichées que l'on n'est pas près d'épuiser, il convient de souligner le dessein du poète. Et là c'est la démarche même de Prométhée, le rival des Dieux, qui se manifeste : dans Le Prologue de la première fable du Livre V, Le Bûcheron et Mercure, elle se lit dans la vision ramassée, véritable «mise en abyme » topique de l'œuvre :

Tantôt je peins en un récit

La sotte vanité jointe avecque l'envie,

Deux pivots sur qui roule aujourd'hui notre vie.

Tel est ce chétif animal

Qui voulut en grosseur au Bœuf se rendre égal.

J'oppose quelquefois, par une double image,

Le vice à la vertu, la sottise au bon sens,

Les Agneaux aux loups ravissants,

La mouche à la Fourmi, faisant de cet ouvrage

Une ample Comédie à cent actes divers,

Et dont la scène est l'Univers.

Suivant l'exemple du héros mythique, qui avait formé l'homme à partir des traits de caractères répartis dans chaque espèce animale, La Fontaine crée un microcosme où les animaux, devenus des "hommes comme les autres ", sont de toutes les époques. Pour dire cet éternel humain, on parle dans le monde des fables simultanément des Dieux, de l'Antiquité, ... et aussi de quelques événements très contemporains.

Le premier recueil des Fables s'était placé dans le sillage d'Esope; l'Avertissement du deuxième annonce avoir cherché « d'autres enrichissements ». Désireux d'introduire de la variété dans son œuvre, La Fontaine déclare maintenant une dette envers Pilpay, un sage indien", et " quelques autres ». En l'absence de source livresque avérée, comme pour la Fable III du Livre VII, Le rat qui s'est retiré du monde, le poète transpose librement l'actualité politique : 
Les Levantins en leur légende

Disent qu'un certain Rat las des soins d'ici-bas,

Dans un fromage de Hollande

Se retira loin du tracas...

«L'ermite nouveau » est bientôt sollicité par des rats venus en délégation lui demander quelques subsides pour Ratopolis assiégée. Alors le « fromage de Hollande » fait entendre une allusion à la guerre de Hollande, très pertinente dans cette fable datée de 1675, année où le clergé régulier avait vivement protesté contre le "don gratuit ", une participation imposée aux dépenses de ladite guerre. Mais dans cette fable nous entendons également une satire, dans la tradition médiévale cette fois, à l'encontre des moines, bien protégés des soucis du siècle, hypocrites, et peu solidaires à l'égard de leurs semblables :

Ayant parlé de cette sorte,

Le nouveau Saint ferma sa porte.

Qui désignai-je à votre avis,

Par ce Rat si peu secourable?

Un moine? Non, mais un Dervis :

Je suppose qu'un Moine est toujours charitable.

Ici un contact s'établit avec l'inspiration drolatique des Contes, la veine gauloise rejoignant la veine galante. C'est donc un aspect moderne des Fables qu'il convient désormais d'interroger.

\section{Un discours moderne. Syncrétisme et pragmatisme}

Les étiquettes sont trompeuses etla posture de La Fontaine dans ses Fables prend en défaut les dichotomies réductrices. On est évidemment plus libre lorsqu'on s'accorde plusieurs maîtres: ce que révèle d'abord le syncrétisme des références rapidement évoqué, c'est la liberté d'esprit du fabuliste, et surtout sa morale qui ne craint pas d'être politiquement incorrecte.

Sous la vulgate d'une polémique abusivement schématisée entre tenants d'une esthétique d'imitation et partisans d'une création émancipée des moules anciens, d'autres enjeux se dessinent. Les Anciens, à l'abri de leurs modèles, et bien adossés à leur monde païen, sont à certains égards plus transgressifs que les Modernes. La Fontaine est d'abord de ceux qui prennent acte de la loi de la nature. Il ne faut pas se le cacher, c'est une dure loi, qui ignore la bienséance, et qui ne ferme pas les yeux sur les pulsions ou le plaisir des sens. De leur côté, les Modernes sont plus assujettis au lissage d'une production littéraire encadrée par les autorités, comme l'Académie et la cour. Marc Fumaroli souligne les positionnements face au pouvoir qui se cachaient sous l'apparent progressisme des Modernes, permettant de mieux comprendre des options à première vue déconcertantes ${ }^{10}$. Ainsi, Boileau, défenseur des Anciens, était un proche de Port-Royal, ce haut lieu du contre-pouvoir du monde des Lettres. La Fontaine était son ami et l'on sait aussi qu'il avait plus d'affinités avec l'esprit des salons parisiens ${ }^{11}$, l'héritage galant de la Fronde, et les penseurs libertins, qu'avec un alignement courtisan tant de fois dénoncé12. Son refus des idées reçues, et un pragmatisme tranquillement provocateur, s'inscrivent dans la topique d'une œuvre qui dit un monde soumis à la raison du plus fort.

Il y a un peu plus d'un demi-siècle André Siegfried, académicien et historien de renom ${ }^{13}$, écrivit un essai intituléLa Fontaine, Machiavel français ${ }^{14}$.Le rapprochement peut surprendre, mais, même s'il appelle de sérieuses mises au point, l'intuition d'une parenté intellectuelle mérite qu'on s'y attarde. On se souvient des griefs de Jean-Jacques Rousseau 
contre ce qu'il appelait "l'immoralité » de La Fontaine ${ }^{15}$. De fait, le fabuliste rejoint le célèbre Florentin reconnu comme un moderne par la postérité ${ }^{16}$, et cela précisément dans sa vision immoraliste du monde. La Fontaine aurait bien compris les leçons de réalisme politique du Prince. André Siegfried commente en ces termes la fable du Lion amoureux: «Samson ne doit pas se laisser couper les cheveux, le lion ne doit pas se laisser rogner les griffes : un Etat désarmé ne compte plus $»^{17}$. La prudence consiste à ne pas croire les discours lénifiants, et à ne pas s'engager sans s'assurer d'un possible retour, le Renard est lucide :

Les pas empreints sur la poussière

Par ceux qui s'en vont faire au malade leur cour,

Tous, sans exception, regardent sa tanière :

Pas un ne marque de retour :

Cela nous met en méfiance.

Que Sa Majesté nous dispense :

Grand merci de son passe-port ;

Je le crois bon ; mais dans cet antre

Je vois fort bien comme l'on entre,

Et ne vois pas comme on en sort. (Le Lion malade et le Renard, VI, 14).

Nous irons plus loin: les victoires ne sont jamais des solutions définitives qui nous permettraient de baisser la garde, et c'est une Iliade en basse-cour qui nous l'enseigne :

Deux Coqs vivaient en paix : une Poule survint

Et voilà la guerre allumée.

Amour, tu perdis Troie : et c'est de toi que vint

Cette querelle envenimée

Où du sang des Dieux même on vit le Xanthe teint!

En effet, le coq vainqueur va chanter sa victoire un peu trop fort :

Un vautour entendit sa voix :

Adieu les amours et la gloire ;

Tout cet orgueil périt sous l'ongle du Vautour.

[...]

Tout vainqueur insolent à sa perte travaille,

Défions-nous du Sort, et prenons garde à nous

Après le gain d'une bataille. (Les Deux coqs, VII, XII)

A nouveau, la référence à l'épopée antique s'inscrit dans un registre héroï-comique qui la désacralise quelque peu. Mythe, distanciation du mythe, et portée transtemporelle, sont interdépendants.

Mais chez La Fontaine, le réalisme politique n'est qu'une application parmi d'autres d'une recommandation générale selon laquelle il faut en toute chose raison garder. Or nos mœurs sont rarement raisonnables... La source de la fable des Deux Chèvres passe pour être une querelle de préséances entre deux dames de haut rang, une certaine Madame de Beringhen et la duchesse de Brissac Saint-Simon qui, s'étant rencontrées dans une rue fort étroite, restèrent, dit-on, cinq heures face à face, faute d'accepter de reculer :

... J'imagine voir avec Louis le Grand

Philippe Quatre qui s'avance

Dans l'île de la Conférence.

Ainsi s'avançaient pas à pas,

Nez à nez, nos Aventurières,

Qui toutes deux étant fort fières,

Vers le milieu du pont ne se voulurent pas

L'une à l'autre céder. Elles avaient la gloire

De compter dans leur race (à ce que dit l'Histoire)

L'une certaine Chèvre au mérite sans pair

Dont Polyphème fit présent à Galatée, 
Et la chèvre Amalthée,

Par qui fut nourri Jupiter.

Faute de reculer, leur chute fut commune;

Toutes deux tombèrent dans l'eau. (Les Deux Chèvres XII, IV)

Les univers de croyances sont ici particulièrement imbriqués : nos chèvres peuvent se prévaloir d'aïeules mythiques, mais leur aventure est celle d'un fait divers contemporain, qui lui-même renvoie ironiquement à un événement diplomatique récent ${ }^{18}$.

En marge des autorités, le pragmatisme de La Fontaine, son refus de l'angélisme, et son regard narquois, sont frappés au coin du bon sens. L'Âne des Animaux malades de la peste, paie cher ses scrupules excessifs. Et bien-sûr, nous répète-t-il souvent,il faut manger pour vivre. Comme nous ne sommes pas tous végétariens, la chaine alimentaire fait que parfois aussi on mange son "prochain »! C'est la leçon du Loup et les Bergers. Le personnage du loup, ou du chat, sont loin d'être toujours négatifs :

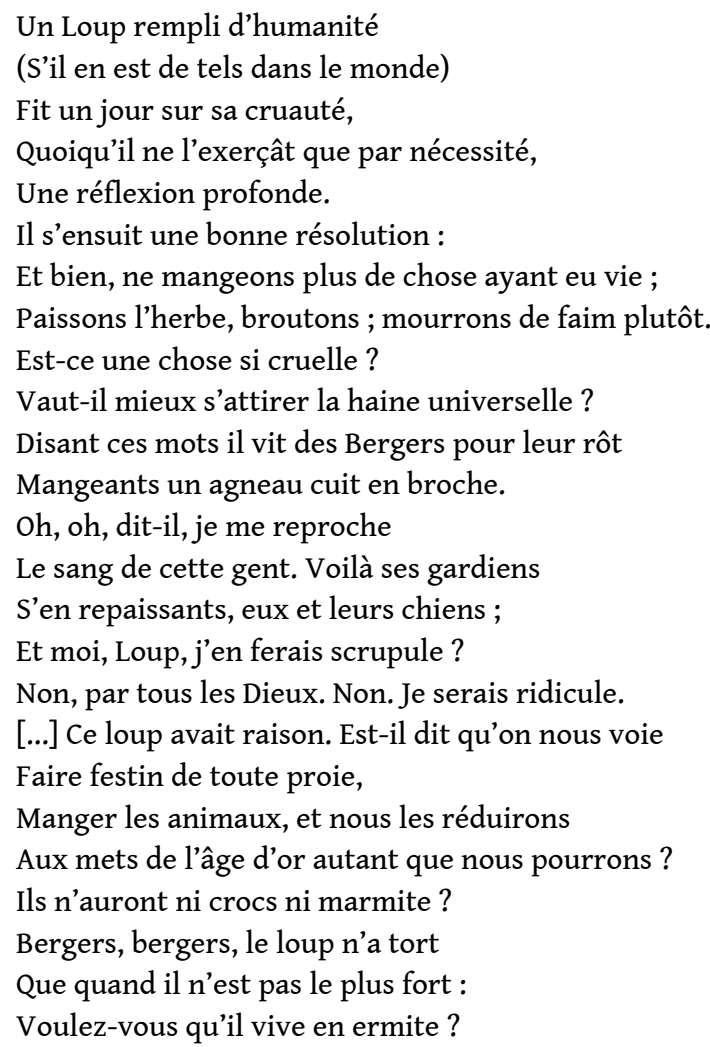

Certaines expressions sont ici révélatrices. Ovide, dans ses Métamorphoses, nous dit en effet que les hommes de l'âge d'or étaient végétariens ${ }^{19}$; les animaux du jardin d'Eden l'étaient aussi, mais, pour La Fontaine, qui en l'occurrence renverrait dos à dos le merveilleux paien comme le merveilleux chrétien, il est clair qu'on ne reviendra pas au Paradis perdu.... Avec une sobre élégance, il récuse l'austérité excessive, et reconnaît une certaine sensualité dans l'acte de se nourririr ${ }^{20}$.

L'allégeance à la loi de la Nature sous-tend un humanisme de la maturité qui s'ouvre à la philosophie d'Epicure, et dépasse les clivages d'école. La nature est changeante et la suivre c'est s'adapter. Comme toujours chez les grands auteurs, entendons ceux dont la réception peut traverser les âges, La Fontaine fait converger éthique et esthétique.Le modèle de souplesse et d'adaptation que nous offre la nature trouve une traduction dans la forme. La fable devient sous sa plume un genre qui ne cesse de se métamorphoser : elle est fondée à s'assumer ainsi, car la pensée ne saurait se couler dans un moule unique. 
Fiction et diction progressent ensemble: une autre modernité de La Fontaine, souvent remarquée des linguistes, se comprend ainsi. Ainsi la remarquable hétérogénéité énonciative, ce concert des voix qui se signale dans le discours des Fables, sera mis en relation avec la pensée plurielle très intégrée qui les caractérise. Les formes libres de discours rapporté sont ici le fer de lance d'une locution polyphonique audacieuse, très en avance sur son temps. On ne reviendra pas sur des démonstrations faites antérieurement ${ }^{21}$, mais il convenait de souligner une fois de plus la cohérence du dit et du dire, du contenu et de la forme.

L'imitation servile des Anciens est donc bien loin en effet. La maîtrise du discours permet de dire une vérité de soi, et manifestement la sagesse de La Fontaine postule un ailleurs. A l'écart des allées du pouvoir assurément (même si, comme tous les auteurs de son temps, il doit solliciter la protection d'un Grand du Royaume), en un lieu où il affirme son inaliénable liberté, et sa propre hiérarchie des valeurs : "Deux vrais amis vivaient au Monomotapa... ». Le réalisme fait place à l'utopie, et, en tout état de cause, à la primauté des sentiments :

Deux vrais amis vivaient au Monomotapa :

L'un ne possédait rien qui n'appartînt à l'autre :

Les amis de ce pays-là

Valent bien dit-on ceux du nôtre. (Les deux Amis, VIII, XI)

L'anecdote montre ensuite ces deux amis faisant assaut de dévouement et de délicatesse. Au moment de l'arrestation de Fouquet en 1661, et de son procès, en 1664, La Fontaine lui était resté fidèle, et cette fidélité lui avait valu l'ordre de s'exiler quelques temps dans le Limousin. C'est cette liberté de l'esprit qui fonde sa posture éthique, lui permettant de dépasser une "Querelle» de principes, et de promouvoir deux cultures parfaitement compatibles : aux côtés de l'innutrition antique, l'exigence d'une morale « galante » qui, dans une société choisie, mettait au premier plan la délicatesse de l'esprit et du cœur, la lucidité et la tendresse des sentiments, l'excellence de l'amitié.

L'humanisme de La Fontaine implique la qualité de l'humain : ce sont les deux faces d'un même signe. Un signe des plus congruents pour l'hommage que je tiens à rendre à Chantal Kircher, à sa personne, et à son rayonnement dans l'université.

\section{BIBLIOGRAPHIE}

Chauveau, Jean-Pierre, 1995, « Le Pouvoir des Fables ou la royauté de La Fontaine », Rumeur des Âges, Commémoration du $3^{\mathrm{e}}$ centenaire de la mort de J. de La Fontaine.

Collinet, Jean-Pierre, 1991, Fables et Contes, O.C., Paris, Gallimard.DANDREY, Patrick, 1991, La Fabrique des Fables. Essai sur la poétique de La Fontaine, Paris, Klincksieck.

Dandrey, Patrick, 1995, La Fontaine ou les Métamorphoses d'Orphée, Paris, Gallimard.

Fumaroli, Marc, 1998, La Diplomatie de l'esprit : de Montaigne à La Fontaine, Paris, Herman.

Fumaroli, Marc, 2001, La Querelle des Anciens et des Modernes, illustrée d'extraits, Paris, GallimardFolio. 
Jaubert, Anna, 1997 : « Labyrinthes énonciatifs », Modèles linguistiques t. XVIII, fasc. 1, p. 17-31. Jaubert, Anna, $2000 \mathrm{a}:$ : Tendre chair et dent dure dans le creuset des Fables deLa Fontaine », Nourritures et écriture (dir. M.-H. Cotoni), T. II, Publications de la Faculté des Lettres de Nice, pp. 97-110.

Jaubert, Anna, $2000 \mathrm{~b}:$ : Le discours indirect libre : dire et montrer. Approche pragmatique », Le style indirect libre et ses contextes, Cahiers Chronos 5, Amsterdam, Rodopi, 2000, p. 49-69.

Jaubert, Anna, 2002 : « Énonciation clivée et discours littéraire. La pragmatique à large spectre des vrais et faux reports de voix », Pragmatique et analyse des textes, Amossy R. (éd.), Tel Aviv, Tel Aviv University, p. 61-93.

Lecoq, Anne-Marie, 2001, La Querelle des Anciens et des Modernes : XVII ${ }^{\mathrm{e}}$-XVIII ${ }^{\mathrm{e}}$ siècle, Paris: Gallimard.

Niderst, Alain, 1987, « Les « Gens de Paris » et les « Gens de Versailles » dans Louise Godard de Donville », D'un siècle à l'autre : anciens et modernes, Paris, Éd. du CNRS, 1987 (Centre Méridional de Rencontres sur le XVII siècle) pp. 159-165.

Sancier-Château, Anne, « Modernité de La Fontaine », Présence de La Fontaine, U. Jean Moulin, Lyon, 1995.

Siegfried, André, La Fontaine, Machiavel français, Paris Ventadour, 1955.

Valéry, Paul, La Fontaine, Paris, Fayard, 1954.

\section{NOTES}

1. Mais qui s'enracine en amont et se prolonge en aval recoupant des enjeux plus profonds sur le sens de la culture, et l'émancipation d'une « autorité ".

2. A Monseigneur L'Evêque de Soissons, La Fontaine, Euvres diverses, (éd. P. Clarac), Paris, Gallimard, Pléiade, 1958, p. 647-649.

3. A Monseigneur le Dauphin, Fables (éd. G. Couton, Garnier-Frères, Paris, 1962), p. 31

4. Préface au premier recueil des Fables (op.cit, p. 5).

5. La Tortue et les deux Canards, X, II. C'est moi qui souligne.

6. «L'art de la transition» que Leo Spitzer saluait chez La Fontaine se déploie aussi dans cette façon de se mouvoir d'un « univers de croyance » à un autre.

7. L'univers de croyance est l'ensemble des propositions implicites tenues pour vraies par un locuteur au moment où il s'exprime. Cet ensemble représente la toile de fond de la cohérence discursive.

8. Il se trouve que Peau d'âne fait partie de ces contes transmis par la tradition populaire, que justement Perrault (le Moderne !) immortalisera dans la forme écrite que l'on sait.

9. Dont certains, entraînés par leur préjugé, supposaient qu'il était Esope lui-même connu sous le nom de Lockman.

10. Marc Fumaroli, La Querelle des Anciens et des Modernes,a illustrée d'extraits, Paris, GallimardFolio, 2001.

11. Il fut longtemps «l'homme de lettres » de Madame de la Sablière, et dans son salon il avait noué des relations avec Bernier, le vulgarisateur de Gassendi.

12. Citons entre autres : Les Animaux malades de la peste, Les obsèques de la Lionne, La Cour du lion...

13. Il est un des pères de l'histoire politique contemporaine.

14. Paris, Ventadour, 1955. 
15. Dans son Emile ou de l'Education, il considérait que les Fables encourageaient moins à se corriger sur la dupe qu'à se former sur le fripon (J.-J. Rousseau, Emile, Livre II, Paris, GF-Flammarion, p. 140-145).

16. Les historiens reconnaissent Machiavel comme un des fondateurs de la pensée politique moderne (voir Quentin Skinner, Les fondements de la pensée politique moderne, Paris, Albin Michel, Bibliothèque de l'Evolution de l'Humanité, 2001.)

17. Le Lion amoureux, IV, I. La réflexion d'André Siegfried est fortement imprégnée par le souvenir très frais de la seconde guerre mondiale : en l'occurrence, se profile l'arrièreplan de l'évacuation prématurée de la rive gauche du Rhin, décidée en Conseil le 9 mars 1936 (A. Siegfried, Introduction à l'ouvrage cité).

18. C'est sur l'île de la Conférence ou île des Faisans, au milieu de la Bidassoa (en pays basque), que les rois de France et d'Espagne s'étaient rencontrés pour le mariage de Louis XIV avec l'infante Marie-Thérèse, et que fut signée en 1659 la paix des Pyrénées.

19. Ovide, Métamorphoses, I., 103

20. Voir Jaubert 2000a

21. Voir Jaubert 1997, 2000 b, 2002.

\section{RÉSUMÉS}

Lors de la Querelle des Anciens et des Modernes, La Fontaine avait dans sa célèbre Epitre à Huet (1687) affiché sa position en faveur des Anciens, mais en même temps il prenait soin de déclarer «mon imitation n'est point un esclavage». A juste titre. La critique a souvent insisté sur sa « modernité ». L'étude qui suit rappellera quelques traits significatifs de cette modernité. Mais il faut garder à l'esprit les enjeux idéologiques de l'époque. Dans ce contexte La Fontaine proclame avant tout son indépendance d'esprit. Il prouve cette indépendance en s'appropriant et en transformant l'héritage des Anciens, devenu avec lui une référence libératrice.

At the time of the Quarrel beetween the Ancients and the Moderns, La Fontaine pronounced himself in favour of the Ancients in his famous Epitre à Huet (1687). But in the same time he attentively proclaimed "mon imitation n'est point un esclavage" ("my imitation is not a servitude"). It was true : literary criticism has often dwelled on his "modernness". This paper will remind some significant features of this modernness. But we have to keep in mind the ideological issues of this historical context. La Fontaine is above all a freethinker. He proved his independence in appropriating to his use the Ancients inheritance and transforming it, making this relation a liberating one.

\section{INDEX}

Keywords : adaptation, free mind, humanism, imitation, La Fontaine, quarrel between the ancients and the moderns, sense of culture, syncretism

Mots-clés : adaptation, esprit libre, humanisme, imitation, querelle des Anciens et des Modernes, sens de la culture, syncrétisme 
AUTEUR

ANNA JAUBERT

Université de Nice-Sophia Antipolis. BCL/CNRS/MSH 